\title{
Correction: Genetic data and cognitively defined late-onset Alzheimer's disease subgroups
}

\author{
Shubhabrata Mukherjee ${ }^{1}$ - Jesse Mez $^{2}$ • Emily H. Trittschuh ${ }^{3,4}$. Andrew J. Saykin $\mathbb{1}^{5} \cdot$ Laura E. Gibbons $^{1}$. \\ David W. Fardo ${ }^{6} \cdot$ Madeline Wessels $^{7}$. Julianna Bauman ${ }^{7} \cdot$ Mackenzie Moore $^{7}$ - Seo-Eun Choi ${ }^{1}$ - Alden L. Gross ${ }^{8}$. \\ Joanne Rich ${ }^{9} \cdot$ Diana K. N. Louden ${ }^{9}{ }^{9} \cdot$ R. Elizabeth Sanders ${ }^{1} \cdot$ Thomas J. Grabowski $^{10,11} \cdot$ Thomas D. Bird $^{4,10}$. \\ Susan M. McCurry ${ }^{12}$ - Beth E. Snitz ${ }^{13} \cdot$ M. Ilyas Kamboh ${ }^{14}$. Oscar L. Lopez ${ }^{13,15}$. Philip L. De Jager ${ }^{16}$. \\ David A. Bennett ${ }^{17}$. C. Dirk Keene ${ }^{18} \cdot$ Eric B. Larson ${ }^{1,19}$ - EPAD Study Group - Investigators from ACT • \\ Investigators from ROS · Investigators from MAP • Investigators from ADNI · Investigators from \\ the University of Pittsburgh ADRC - Paul K. Crane ${ }^{1}$
}

Published online: 15 January 2019

(c) The Author(s) 2019. This article is published with open access

\section{Correction to: Molecular Psychiatry; https://doi.org/10.1038/s41380-018-0298-8; published online 04 December 2018}

This article was originally published under standard licence, but has now been made available under a [CC BY 4.0] license. The PDF and HTML versions of the paper have been modified accordingly.

Paul K. Crane

pcrane@uw.edu

1 Department of Medicine, School of Medicine, University of Washington, Seattle, WA, USA

2 Department of Neurology, Boston University School of Medicine, Boston, MA, USA

3 Department of Psychiatry and Behavioral Sciences, School of Medicine, University of Washington, Seattle, WA, USA

4 VA Puget Sound Health Care System, Seattle, WA, USA

5 Department of Radiology, Indiana University, Indianapolis, IN, USA

6 Department of Biostatistics, University of Kentucky, Lexington, KY, USA

7 College of Arts and Sciences, School of Nursing, University of Washington, Seattle, WA, USA

8 Department of Epidemiology, Johns Hopkins Bloomberg School of Public Health, Baltimore, MD, USA

9 Health Sciences Library, University Libraries, University of Washington, Seattle, WA, USA

10 Department of Neurology, School of Medicine, University of
Open Access This article is licensed under a Creative Commons Attribution 4.0 International License, which permits use, sharing, adaptation, distribution and reproduction in any medium or format, as long as you give appropriate credit to the original author(s) and the source, provide a link to the Creative Commons license, and indicate if changes were made. The images or other third party material in this article are included in the article's Creative Commons license, unless indicated otherwise in a credit line to the material. If material is not included in the article's Creative Commons license and your intended use is not permitted by statutory regulation or exceeds the permitted use, you will need to obtain permission directly from the copyright holder. To view a copy of this license, visit http://creativecommons. org/licenses/by/4.0/.

Washington, Seattle, WA, USA

11 Department of Radiology, School of Medicine, University of Washington, Seattle, WA, USA

12 Department of Psychosocial and Community Health, School of Nursing, University of Washington, Seattle, WA, USA

13 Department of Neurology, University of Pittsburgh, Pittsburgh, PA, USA

14 Departments of Human Genetics, University of Pittsburgh, Pittsburgh, PA, USA

15 Departments of Psychiatry, University of Pittsburgh, Pittsburgh, PA, USA

16 Center for Translational \& Computational Neuroimmunology, Department of Neurology, Columbia University Medical Center, New York, NY, USA

17 Rush Alzheimer's Disease Center, Rush University Medical Center, Chicago, IL, USA

18 Department of Pathology, School of Medicine, The University of Washington, Seattle, WA, USA

19 Kaiser Permanente Washington Health Research Institute, Seattle, WA, USA 